\title{
COMPARISON OF DIFFERENT APPROXIMATIONS OF FUZZY NUMBERS
}

\author{
D. Stephen Dinagar ${ }^{1}$ and K.Jivagan ${ }^{2}$ \\ ${ }^{1,2} \mathrm{PG}$ and Research Department of Mathematics, T.B.M.L. College, Porayar, India.
}

\begin{abstract}
The notions of interval approximations of fuzzy numbers and trapezoidal approximations of fuzzy numbers have been discussed. Comparisons have been made between the close-interval approximation, valueambiguity interval approximation and distinct approximation with the corresponding crisp and trapezoidal fuzzy numbers. A numerical example is included to justify the above mentioned notions.
\end{abstract}

\section{KEYWORDS}

Close interval approximations, value ambiguity interval approximations, Distinct approximations.

\section{INTRODUCTION}

The game theory is widely applied to military affairs, physical training, and commercial production and so on. But the classical game theory is based on the double value logic theory lose sight of much fuzzy information and grey information.

A game is a description of a decision-making situation involving more than one decision maker. Game theory is generally considered to have begun with the publication of von Neumann and Morgenstern's The Theory of Games and Economic Behavior [1,13] in 1944. The development of game theory accelerated in papers by Nash [5,6], Shapley [7], [8], and Gillies [4]. The behavior of players in a game is assumed to be rational and influenced by other rational players' behaviors, which distinguishes a game from the general decision-making problem.

In order to analyze the behaviors of players and construct a method for each player to choose his action, a strategic game first defines each individual's alternative actions. The combination of all the players' strategies will determine a unique outcome to the game and the payoffs to all players.

A solution for a player in a game should allow that player to win or satisfy his objectives for the game. For example, he can maximize his own payoff and/or minimize his opponent's payoff. Formally, the solution of a game is a situation in which each player plays a best response to the other players' actual strategy choices. This is the concept of equilibrium.

There are several methods for obtaining the equilibrium of some special kinds of games such as the dominant strategy for dominant strategy equilibrium or a mixed strategy for mixed strategy equilibrium. A linear programming method is used in matrix games. Most of these methods are based on the maximin principle for selecting optimal strategies. But in 1978, Butnariu [2] pointed out that one of the major assumptions of classical game theory is that all possible choosing strategies are equally possible choices for a player.

DOI : $10.5121 /$ ijfls.2015.5402 
The notion of fuzzy sets first appeared in the papers written by Zadeh $[14,15]$. This notion tries to show that an object more or less corresponds to a particular category. The degree to which an element belongs to a category is an element of the continuous interval $[0,1]$ rather than the Boolean pair $\{0,1\}$ [15]. Using the notion of fuzzy sets, the payoff function in a game can be fuzzified. Furthermore, the solution to the game may also be a fuzzy set.

The paper is organized as follows: The section 1 gives introduction is introductory in nature. Section 2 gives the basic definitions and concepts needed for this work. In section 3, the comparisons of different approximations in 'fuzzy game notion' have been presented. In section 4 , the comparisons of different approximations in two person zero-sum method in fuzzy environment have been discussed. In section 5, a relevant numerical example to compare the different approximations of fuzzy numbers to justify the above discussed notions are included. The concluding remarks are also added in the last section.

\section{Preliminaries}

\subsection{Definition (Fuzzy Set [FS])}

Let $X$ denote a universal set i.e., $X=\{x\}$; then the characteristic function which assigns certain values or a membership grade to the elements of this universal set within a specified range $\{0,1\}$ is known as the membership function and the set thus defined is called a fuzzy set. The membership graders correspond to the degree to which an element is compatible with the concept represented by the fuzzy set. If $\mu_{\tilde{A}}$ is the membership function defining fuzzy set $\tilde{A}$, then,

$\mu_{\tilde{\mathrm{A}}}: X \rightarrow[0,1]$, where $[0,1]$ developed the interval of real numbers from 0 to 1 .

\subsection{Definition (Fuzzy Number $[\mathrm{FN}]$ )}

A convex and normalized fuzzy set defined on R. whose membership function is piecewise continuous is called fuzzy number. A fuzzy set is called normal when at least one of its elements attains the maximum possible membership grade.

$$
\text { i.e., } \max _{x} \mu_{A}(x)=1, \forall \mathrm{x} \in \mathrm{R}
$$

\subsection{Definition ( $\alpha$-CUT)}

An $\alpha$-cut of a fuzzy set $\tilde{\mathrm{A}}$ is a crisp set $\tilde{\mathrm{A}}_{\alpha}$ that contains all the elements of the universal set $\mathrm{X}$ that have a membership grade in A greater than or equal to the specified value of a. Thus,

$$
\tilde{\mathrm{A}}_{\alpha}=\left\{\mathrm{x} \in X ; \mu_{\tilde{A}}(x) \geq \alpha, 0 \leq x \leq 1\right\}
$$

\subsection{Definition (Close Interval Approximation of Trapezoidal Fuzzy Numbers [CIATFN]) [11]}

Let $\tilde{A}=\left(a_{1}, a_{2}, a_{3}, a_{4}\right)$ be a trapezoidal fuzzy number, and its interval approximation of fuzzy number $[\tilde{A}]=\left[a_{\alpha}^{L}, a_{\alpha}^{U}\right]$ is said to be close interval approximation if

$$
\begin{aligned}
& a_{L}=\inf \left\{x \in A / \mu_{A}(x) \geq 0.5\right\}=\frac{a_{1}+a_{2}}{2} \\
& a_{U}=\operatorname{Sup}\left\{x \in A / \mu_{A}(x) \geq 0.5\right\}=\frac{a_{3}+a_{4}}{2}
\end{aligned}
$$




\subsection{Definition (Value of a Fuzzy Number [VFN]) [9]}

Let $\tilde{\mathrm{A}}$ be a fuzzy number with $\alpha$-cut representation $\left(A_{L}(\alpha), A_{U}(\alpha)\right)$, then the value of $\tilde{\mathrm{A}}$ is defined as

$$
\operatorname{Val}(\tilde{\mathrm{A}})=\int_{0}^{1} \alpha\left[A_{U}(\alpha)+A_{L}(\alpha)\right] d \alpha
$$

\subsection{Definition (Ambiguity of a Fuzzy Number [AFN]) [9]}

Let $\tilde{\mathrm{A}}$ be a fuzzy number with $\alpha$-cut representation $\left(A_{L}(\alpha), A_{U}(\alpha)\right)$, then the ambiguity of $\tilde{\mathrm{A}}$ is defined as

$$
\operatorname{Amb}(\tilde{\mathrm{A}})=\int_{0}^{1} \alpha\left[A_{U}(\alpha)-A_{L}(\alpha)\right] d \alpha
$$

\subsection{Definition (Value-Ambiguity Interval Approximation of Fuzzy Number [VAIA])[10]}

An interval approximation operator $V_{A}$ is defined as

$V_{A}: \mathrm{F}(\mathrm{R}) \rightarrow F^{I}(R)$ in a way that $V_{A}: \tilde{\mathrm{A}} \rightarrow V_{A}(\tilde{\mathrm{A}})=\left[a^{-}, a^{+}\right]$

$$
\begin{aligned}
& \text { where } a^{-}=2 \int_{0}^{1} \alpha\left(A_{L}(\alpha)\right) d \alpha \\
& a^{+}=2 \int_{0}^{1} \alpha\left(A_{U}(\alpha)\right) d \alpha
\end{aligned}
$$

Then the operator $V_{A}$ is called a value-ambiguity interval approximation operator, Also the interval $\left[a^{-}, a^{+}\right]$is called the value - ambiguity interval approximation of $\tilde{\mathrm{A}}$.

\subsection{Definition (Distinct Approximation of Trapezoidal Fuzzy Number [DATrFN]) [12]}

Let $\tilde{A}^{\prime}=\left(a_{1}^{\prime}, a_{2}^{\prime}, a_{3}^{\prime}, a_{4}^{\prime}\right)$ be a distinct approximation of $\tilde{A}=\left(a_{1}, a_{2}, a_{3}, a_{4}\right)$. where $a_{1}^{\prime}=\frac{3 a_{1}-a_{2}}{2}$, $a_{2}^{\prime}=\frac{a_{1}+a_{2}}{2}, \quad a_{3}^{\prime}=\frac{a_{4}+a_{3}}{2}, a_{4}^{\prime}=\frac{3 a_{4}-a_{3}}{2}$

Then the membership function of DATrFN is

$$
\mu_{\tilde{A}^{\prime}}(x)=\left\{\begin{array}{cl}
\frac{2 x-\left(a_{2}+a_{1}\right)}{2\left(a_{2}-a_{1}\right)} & \text { if } \frac{a_{1}+a_{2}}{2} \leq x<\frac{3 \mathrm{a}_{1}-a_{2}}{2} \\
1 & \text { if } \frac{a_{3}+a_{4}}{2} \leq x \leq \frac{a_{1}+a_{2}}{2} \\
\frac{\left(a_{3}+a_{4}\right)-2 x}{2\left(a_{4}-a_{3}\right)} & \text { if } \frac{3 a_{4}-a_{3}}{2}<x \leq \frac{a_{3}+a_{4}}{2} \\
0 & \text { otherwise }
\end{array}\right.
$$


International Journal of Fuzzy Logic Systems (IJFLS) Vol.5, No.4, October 2015

\subsection{Definition (Associated Real Value of Approximation of Interval Fuzzy Number)}

If $[\tilde{A}]=\left[t_{1}, t_{2}\right]$ is an close interval approximation of trapezoidal fuzzy number $\tilde{A}=\left(a_{1}, a_{2}, a_{3}, a_{4}\right)$, then its associated real value is given by

$$
\hat{A}=\frac{\left[t_{1}+t_{2}\right]}{2}
$$

\subsection{Definition (Associated Real Value of Trapezoidal Fuzzy Number)}

If $\tilde{A}=\left(a_{1}, a_{2}, a_{3}, a_{4}\right)$ is a trapezoidal fuzzy number, then its associated real value is given by

$$
\hat{A}=\frac{\left(a_{1}+a_{2}+a_{3}+a_{4}\right)}{4}
$$

\subsection{Definition (Ranking of Fuzzy Numbers [RFN])}

Let $\tilde{A}$ and $\tilde{B}$ be any two fuzzy numbers. Then $\tilde{A}$ is greater than $\tilde{B}$ if and only if $\hat{A} \succ \hat{B}$ and denoted by $\tilde{A} \succ \widetilde{B}$.

\subsection{Definition (Maximum of Fuzzy Numbers)}

Let $\tilde{A}$ and $\tilde{B}$ be any two fuzzy numbers. Then the maximum value of $\tilde{A}$ and $\tilde{B}$ denoted by $\operatorname{Max}(\tilde{A}, \tilde{B})$ and is defined by $\operatorname{Max}(\tilde{A}, \tilde{B})=\tilde{A}$, if $\tilde{A} \succ \tilde{B}$.

\subsection{Definition (Minimum of Fuzzy Numbers)}

Let $\tilde{A}$ and $\widetilde{B}$ be any two fuzzy numbers. Then the minimum value of $\tilde{A}$ and $\widetilde{B}$ denoted by $\operatorname{Min}(\tilde{A}, \tilde{B})$ and is defined by $\operatorname{Min}(\tilde{A}, \tilde{B})=\tilde{A}$, if $\tilde{A} \prec \tilde{B}$.

\subsection{Definition (Arithmetic Operations of Interval Fuzzy Numbers)}

Let $\tilde{A}=\left[a_{1}, a_{2}\right], \tilde{B}=\left[b_{1}, b_{2}\right], \forall a_{1}, a_{2}, b_{1}, b_{2} \in R$, be any two interval fuzzy numbers. Then

Addition:

$\left[a_{1}, a_{2}\right](+)\left[b_{1}, b_{2}\right]=\left[a_{1}+b_{1}, a_{2}+b_{2}\right]$

\section{Subtraction:}

$\left[a_{1}, a_{2}\right](-)\left[b_{1}, b_{2}\right]=\left[a_{1}-b_{2}, a_{2}-b_{1}\right]$

\section{Multiplication:}

$$
\left[a_{1}, a_{2}\right](\bullet)\left[b_{1}, b_{2}\right]=\left[a_{1} \bullet b_{2}, a_{2} \bullet b_{1}\right]
$$




\subsection{Arithmetic Operations of Trapezoidal Fuzzy Numbers}

Let $\tilde{A}=\left(a_{1}, a_{2}, a_{3}, a_{4}\right)$ and $\tilde{B}=\left(b_{1}, b_{2}, b_{3}, b_{4}\right)$ be any two trapezoidal fuzzy numbers. Then

Addition:

$A(+) B=\left(a_{1}+b_{1}, a_{2}+b_{2}, a_{3}+b_{3}, a_{4}+b_{4}\right)$

\section{Subtraction:}

$A(-) B=\left(a_{1}-b_{4}, a_{2}-b_{3}, a_{3}-b_{2}, a_{4}-b_{1}\right)$

\section{Multiplication:}

$$
\begin{aligned}
A(\bullet) B & =\left(a_{1}, a_{2}, a_{3}, a_{4}\right)(\bullet)\left(b_{1}, b_{2}, b_{3}, b_{4}\right) \\
& =\left(a_{1} b_{1}, a_{2} b_{2}, a_{3} b_{3}, a_{4} b_{4}\right)
\end{aligned}
$$

\section{FuZzY APPROACH To STRATEgic GAMES}

A strategy is a complete plan for a player to decide how to play the game [3]. Without detailed knowledge of the sequence of moves in a strategic game, we can still analyze the behaviors of players; all that is needed for a solution to a game is to indicate what the player would do in the situation when he must make a move. So it is sufficient if we list each individual's strategies, the combination of which will determine a unique outcome (payoff to each player) of a game. A game is in strategic form if only the set of players I, the set of strategies $S_{i}$ and the payoff functions $P_{i}(s)$ for each player $i \in I$ are given, where $S=\left(S_{1}, S_{2}, \ldots . . S_{I}\right)$. The concepts of games in strategic form and mixed strategies used in this paper, as well as the notations, are as defined and described in [3].

\subsection{Methodology of Comparison of Different Approximations by Using Two- Person Zero Sum in Existing Saddle Point Method in Fuzzy Environment}

The fuzzy game with two players were the gain of one player is equal to the loss of the other player is called two person 'zero' sum game. The resulting gain or loss for the player ' $A$ ' in a game between players ' $\mathrm{A}$ ' and ' $\mathrm{B}$ ' can be represented in the form of a matrix which is called the payoff matrix. In each entry are fuzzy numbers. To obtain the solution of the game we can use the Minimax- Maximin principle. Using which we find the fuzzy saddle point.

Using this fuzzy saddle point, the value of the game is obtained and this saddle point has a corresponding strategy for player 'A' and 'B'. If a saddle point exists then the strategy follows by pure-strategy. (If fuzzy saddle point does not exist then we have mixed strategy). The value of the game which corresponding to the saddle point represents the Maximum guaranteed gain for player 'A'.

(i.e.,) Guaranteed

(i.e.,) Maximum possible loss for player 'B' 
The value of the fuzzy game is used to represent ' $\widetilde{V}$ ' moreover if a saddle point exists then the game is said to be strictly determined.

In general for any given fuzzy game the maximin value is denoted by ' $\underline{V}$ ' and minimax value is denoted by ' $\overline{\widetilde{V}}$ ' for any fuzzy game the following inequalities is

However if a fuzzy saddle point exists.

$$
\underline{\tilde{V}} \leq \tilde{V} \leq \overline{\tilde{V}} \text { if and only if }
$$

$$
\underline{\tilde{V}}=\tilde{V}=\overline{\tilde{V}}
$$

If the value of the game $\tilde{V}=\tilde{0}$ that such a fuzzy game is said to be fair game.

\section{Steps to find the fuzzy saddle point}

\section{Step: 1}

Find the minimum variable of each row of the payoff matrix and mark them.

\section{Step: 2}

Find the Maximum variable of each column of the payoff matrix and mark them.

\section{Step: 3}

If in this process then there exists a point in the payoff matrix with the both above marks. Then this point corresponds to the saddle point. Using this value of saddle point is found. Using which corresponding pure strategies for players ' $\mathrm{A}$ ' and ' $\mathrm{B}$ ' are found.

\subsection{Close-Interval Approximation of Trapezoidal Fuzzy Numbers (CIATrFN)}

Let $\tilde{A}=\left(a_{1}, a_{2}, a_{3}, a_{4}\right), \tilde{B}=\left(b_{1}, b_{2}, b_{3}, b_{4}\right), \tilde{C}=\left(c_{1}, c_{2}, c_{3}, c_{4}\right)$ and $\tilde{D}=\left(d_{1}, d_{2}, d_{3}, d_{4}\right)$ be a trapezoidal fuzzy numbers. Then the close-interval approximations of them given by

$$
\begin{aligned}
C_{D}(\tilde{A}) & =\left[\frac{a_{1}+a_{2}}{2}, \frac{a_{3}+a_{4}}{2}\right], C_{D}(\tilde{B})=\left[\frac{b_{1}+b_{2}}{2}, \frac{b_{3}+b_{4}}{2}\right], C_{D}(\tilde{C})=\left[\frac{c_{1}+c_{2}}{2}, \frac{c_{3}+c_{4}}{2}\right] \text { and } \\
C_{D}(\tilde{D}) & =\left[\frac{d_{1}+d_{2}}{2}, \frac{d_{3}+d_{4}}{2}\right]
\end{aligned}
$$

Now, solve the fuzzy game whose payoff matrix is given by

$$
\begin{array}{cc}
\text { Player B } & \text { Min } \\
\text { Player A } & {\left[\begin{array}{cc}
{\left[\frac{a_{1}+a_{2}}{2}, \frac{a_{3}+a_{4}}{2}\right]} & {\left[\frac{b_{1}+b_{2}}{2}, \frac{b_{3}+b_{4}}{2}\right]} \\
\operatorname{Max} & {\left[\frac{c_{1}+c_{2}}{2}, \frac{c_{3}+c_{4}}{2}\right]} \\
g_{11}(\text { or }) g_{21} & \left.\left[\frac{d_{1}+d_{2}}{2}, \frac{d_{3}+d_{4}}{2}\right]\right] g_{11}(\text { or }) g_{12} \\
g_{12}(\text { or }) g_{22}
\end{array}\right] g_{21}(\text { or }) g_{22}}
\end{array}
$$

If Minimax = Maximin,

Therefore the saddle point is exists. Then we can find the value of the fuzzy game $(\tilde{V})$. And then we can find the Strategy of player A. i.e., $S_{A}$ and Strategy of player B. i.e., $S_{B}$ 


\subsection{Value-Ambiguity Interval Approximation of Trapezoidal Fuzzy Numbers (VAIA)}

Let $\tilde{A}=\left(a_{1}, a_{2}, a_{3}, a_{4}\right) ; \tilde{B}=\left(b_{1}, b_{2}, b_{3}, b_{4}\right) ; \tilde{C}=\left(c_{1}, c_{2}, c_{3}, c_{4}\right)$ and $\tilde{D}=\left(d_{1}, d_{2}, d_{3}, d_{4}\right)$ are the trapezoidal fuzzy numbers. Then the value of the fuzzy number $\tilde{A}$ is

i.e., $\operatorname{Val}(\tilde{A})=\frac{a_{4}+2\left(a_{3}+a_{2}\right)+a_{1}}{6}$;

and similarly for

$\operatorname{Val}(\tilde{B})=\frac{b_{4}+2\left(b_{3}+b_{2}\right)+b_{1}}{6} ; \quad \operatorname{Val}(\tilde{C})=\frac{c_{4}+2\left(c_{3}+c_{2}\right)+c_{1}}{6} ;$

$\operatorname{Val}(\tilde{D})=\frac{d_{4}+2\left(d_{3}+d_{2}\right)+d_{1}}{6}$

and the Ambiguity of fuzzy number $\tilde{A}$ is

i.e., $\operatorname{Amb}(\tilde{A})=\frac{a_{4}+2\left(a_{3}-a_{2}\right)-a_{1}}{6}$;

and similarly for

$$
\begin{array}{ll}
A m b(\tilde{B})=\frac{b_{4}+2\left(b_{3}-b_{2}\right)-b_{1}}{6} ; & A m b(\tilde{C})=\frac{c_{4}+2\left(c_{3}-c_{2}\right)-c_{1}}{6} ; \\
A m b(\tilde{D})=\frac{d_{4}+2\left(d_{3}-d_{2}\right)-d_{1}}{6} &
\end{array}
$$

Therefore the Value-Ambiguity interval approximations of trapezoidal fuzzy number $\tilde{A}$ is denoted by $V_{A}(\tilde{A})$.

$$
V_{A}(\tilde{A})=[\operatorname{Val}(\tilde{A})-A m b(\tilde{A}), \operatorname{Val}(\tilde{A})+A m b(\tilde{A})]=\left[\frac{a_{1}+2 a_{2}}{3}, \frac{a_{4}+2 a_{3}}{3}\right],
$$

Similarly

$$
\begin{aligned}
& V_{A}(\tilde{\boldsymbol{B}})=[\operatorname{Val}(\tilde{\boldsymbol{B}})-\operatorname{Amb}(\tilde{\boldsymbol{B}}), \operatorname{Val}(\tilde{\boldsymbol{B}})+\operatorname{Amb}(\tilde{\boldsymbol{B}})]=\left[\frac{b_{1}+2 b_{2}}{3}, \frac{b_{4}+2 b_{3}}{3}\right], \\
& V_{A}(\tilde{\boldsymbol{C}})=[\operatorname{Val}(\tilde{\boldsymbol{C}})-\operatorname{Amb}(\tilde{\boldsymbol{C}}), \operatorname{Val}(\tilde{\boldsymbol{C}})+\operatorname{Amb}(\tilde{\boldsymbol{C}})]=\left[\frac{c_{1}+2 c_{2}}{3}, \frac{c_{4}+2 c_{3}}{3}\right], \\
& V_{A}(\tilde{D})=[\operatorname{Val}(\tilde{D})-\operatorname{Amb}(\tilde{D}), \operatorname{Val}(\tilde{D})+\operatorname{Amb}(\tilde{D})]=\left[\frac{d_{1}+2 d_{2}}{3}, \frac{d_{4}+2 d_{3}}{3}\right] .
\end{aligned}
$$

Now, solve the above fuzzy game whose payoff matrix is given by 
International Journal of Fuzzy Logic Systems (IJFLS) Vol.5, No.4, October 2015

Player B
Player A $\left[\begin{array}{cc}{\left[\frac{a_{1}+2 a_{2}}{3}, \frac{a_{4}+2 a_{3}}{2}\right]} & {\left[\frac{b_{1}+2 b_{2}}{2}, \frac{b_{4}+2 b_{3}}{2}\right]} \\ {\left[\begin{array}{c}\left.\frac{c_{1}+2 c_{2}}{3}, \frac{c_{4}+2 c_{3}}{3}\right] \\ 3\end{array}\right]} & \left.\left[\frac{d_{1}+2 d_{2}}{3}, \frac{d_{4}+2 d_{3}}{3}\right]\right] g_{11}(\text { or }) g_{12} \\ g_{11}(\text { or }) g_{21}\end{array}\right] g_{21}($ or $) g_{22}$

If Minimax = Maximin,

the saddle point is exists. Then we can find the value of the fuzzy game $(\tilde{V})$. And then we can find Strategy of player A. i.e., $S_{A}$ and Strategy of player B. i.e., $S_{B}$.

\subsection{Distinct Approximations of Trapezoidal Fuzzy Numbers (DATFN)}

Let $\tilde{A}^{\prime}=\left(a_{1}^{\prime}, a_{2}^{\prime}, a_{3}^{\prime}, a_{4}^{\prime}\right)$ be a distinct approximation of trapezoidal fuzzy number $\tilde{A}=\left(a_{1}\right.$, $\left.a_{2}, a_{3}, a_{4}\right)$, where $a_{1}^{\prime}=\frac{3 a_{1}-a_{2}}{2}, a_{2}^{\prime}=\frac{a_{1}+a_{2}}{2}, a_{3}^{\prime}=\frac{a_{4}+a_{3}}{2}, a_{4}^{\prime}=\frac{3 a_{4}-a_{3}}{2}$. Distinct approximations of $\tilde{A}$,

$D(\tilde{A})=\left(\frac{3 a_{1}-a_{2}}{2}, \frac{a_{1}+a_{2}}{2}, \frac{a_{3}+a_{4}}{2}, \frac{3 a_{4}-a_{3}}{2}\right)$,

Similarly for

$\widetilde{B}=\left(b_{1}, b_{2}, b_{3}, b_{4}\right)$, then its distinct approximations

$D(\tilde{B})=\left(\frac{3 b_{1}-b_{2}}{2}, \frac{b_{1}+b_{2}}{2}, \frac{b_{3}+b_{4}}{2}, \frac{3 b_{4}-b_{3}}{2}\right)$

$\tilde{C}=\left(c_{1}, c_{2}, c_{3}, c_{4}\right)$ then its distinct approximations

$D(\tilde{C})=\left(\frac{3 c_{1}-c_{2}}{2}, \frac{c_{1}+c_{2}}{2}, \frac{c_{3}+c_{4}}{2}, \frac{3 c_{4}-c_{3}}{2}\right)$

and $\tilde{D}=\left(d_{1}, d_{2}, d_{3}, d_{4}\right)$ then its distinct approximations

$$
D(\tilde{D})=\left(\frac{3 d_{1}-d_{2}}{2}, \frac{d_{1}+d_{2}}{2}, \frac{d_{3}+d_{4}}{2}, \frac{3 d_{4}-d_{3}}{2}\right)
$$

Now, solve the above fuzzy game whose payoff matrix is given by

$$
\begin{gathered}
\text { Player A }\left[\begin{array}{cc}
\left(\frac{3 a_{1}-a_{2}}{2}, \frac{a_{1}+a_{2}}{2}, \frac{a_{3}+a_{4}}{2}, \frac{3 a_{4}-a_{3}}{2}\right) & \left(\frac{3 b_{1}-b_{2}}{2}, \frac{b_{1}+b_{2}}{2}, \frac{b_{3}+b_{4}}{2}, \frac{3 b_{4}-b_{3}}{2}\right) \\
\left(\frac{3 c_{1}-c_{2}}{2}, \frac{c_{1}+c_{2}}{2}, \frac{c_{3}+c_{4}}{2}, \frac{3 c_{4}-c_{3}}{2}\right) & \left(\frac{3 d_{1}-d_{2}}{2}, \frac{d_{1}+d_{2}}{2}, \frac{d_{3}+d_{4}}{2}, \frac{3 d_{4}-d_{3}}{2}\right)
\end{array}\right] g_{11}(\text { or }) g_{12}(\text { or }) g_{22} \\
g_{11}(\text { or }) g_{21}
\end{gathered}
$$

If Minimax $=$ Maximin,

Therefore the saddle point is exists. Then we can find the value of the fuzzy game $(\tilde{V})$. And then we can find Strategy of player A i.e., $S_{A}$ and Strategy of player B i.e., $S_{B}$ 


\section{Mixed Strategy Of Two Person Zero-Sum Method (Without SADDle PoINT) To COMPARE DifFERENT APPROXIMATIONS}

\section{METHODS IN FUZZY ENVIRONMENT}

Consider a fuzzy game whose payoff matrix is of order $2 \times 2$ and which does not have saddle point.

Consider the payoff matrix given by

Player B Min

$$
\begin{aligned}
& \text { Player A }\left[\begin{array}{ll}
a_{11} & a_{12} \\
a_{21} & a_{22}
\end{array}\right] P_{1} \\
& \text { Max } \\
& q_{1} \quad q_{2}
\end{aligned}
$$

If there is no saddle point for the above payoff matrix then,

We know that, mixed strategy is followed. Moreover the value of the game is

$$
\begin{aligned}
& \tilde{V}=\frac{a_{11} a_{22}-a_{12} a_{21}}{\left(a_{11}+a_{22}\right)-\left(a_{12}+a_{21}\right)} \\
& P_{1}=\frac{a_{22}-a_{21}}{\left(a_{11}+a_{22}\right)-\left(a_{12}+a_{21}\right)}
\end{aligned}
$$

We know that,

$$
P_{1}+P_{2}=1 \Rightarrow P_{2}=1-P_{1},
$$

Also,

$q_{1}=\frac{a_{22}-a_{12}}{\left(a_{11}+a_{22}\right)-\left(a_{12}+a_{21}\right)}$ and $q_{1}+q_{2}=1 \Rightarrow q_{2}=1-q_{1}$

\subsection{Example: Game with and without Saddle Point Method in Crisp}

Consider the game

$$
\text { Player A }\left[\begin{array}{cc}
4.7 & 5 \\
5.2 & 7.2
\end{array}\right]
$$

Now, solve the above fuzzy game whose payoff matrix is given by 


\begin{tabular}{|c|c|c|c|}
\hline \multirow{3}{*}{ Player A } & \multicolumn{2}{|c|}{ Player B } & Min \\
\hline & 4.7 & 5 & 4.7 \\
\hline & 5.2 & 7.2 & 5.2 \\
\hline Max & 5.2 & 7.2 & \\
\hline
\end{tabular}

If Minmax $=$ Maximin

Therefore the saddle point is exist. Therefore the value of the fuzzy game $(\tilde{V})=5.2$

The Strategy for player A. i.e., $S_{A}=5$

Strategy for player B. i.e., $S_{B}=7.2$

\subsection{Fuzzy Game with and without Saddle Point Method}

Let $\tilde{A}=(3,4,5,7), \tilde{B}=(2,5,6,7), \tilde{C}=(2,4,6,9), \tilde{D}=(4,7,8,11)$ are the trapezoidal fuzzy numbers. Now, solve the above fuzzy game whose payoff matrix is given by

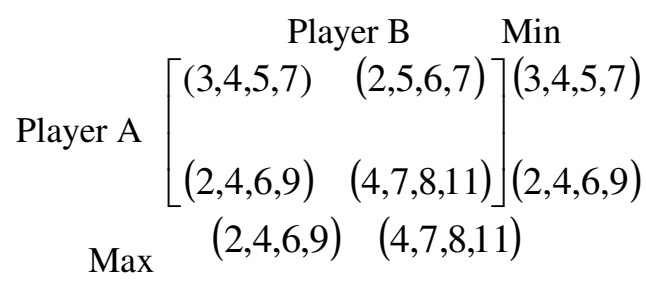

If Minmax = Maximin

Therefore the saddle point is exist.

Therefore the value of the fuzzy game $(\tilde{V})=5.2$.

The Strategy for player A. i.e., $S_{A}=4.7$

Strategy for player B. i.e., $S_{B}=7.2$

\subsection{Close Interval Approximation of Fuzzy Game without Saddle Point Method}

Let $\tilde{A}=(3,4,5,7)$ be a trapezoidal fuzzy number and its close interval approximation is $[\tilde{A}]=[3.5,6]$ (by def 2.4).

Similarly for $\widetilde{B}=(2,5,6,7)$, then its close interval approximation is $[\tilde{B}]=[3.5,6.5]$,

$\tilde{C}=(2,4,6,9)$, then the close interval approximation is $[\tilde{C}]=[3,6.5]$,

and $\tilde{D}=(4,7,8,11)$, then the close interval approximation of $[\tilde{D}]=[5.5,9.5]$.

Now, solve the above fuzzy game whose payoff matrix is given by

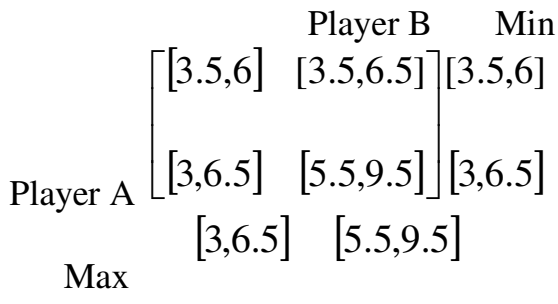


If Minmax $=$ Maximin

Therefore the saddle point is exist.

Therefore the value of the fuzzy game $\tilde{V}=4.7$.

The Strategy for player A. i.e., $S_{A}=[3.5,6]=4.75$

Strategy for player B. i.e., $S_{B}=[5.5,9.5]=7.5$

\subsection{Value-Ambiguity Interval Approximation of Fuzzy Game without Saddle Point Method}

Let $\tilde{A}=(3,4,5,7), \tilde{B}=(2,5,6,7), \tilde{C}=(2,4,6,7), \tilde{D}=(4,7,8,11)$ are the trapezoidal fuzzy numbers and its value ambiguity interval approximation of $\tilde{A}$ is

$\operatorname{Value}(\tilde{A})=\frac{a_{4}+2\left(a_{3}+a_{2}\right)+a_{1}}{6}$,

$\operatorname{Value}(\tilde{A})=\frac{a_{4}+2\left(a_{3}+a_{2}\right)+a_{1}}{6}, \operatorname{Value}(\tilde{B})=5.16, \operatorname{Value}(\tilde{C})=4.8$, and $\operatorname{Value}(\tilde{D})=7.5$.

Also, Ambiguity $(\tilde{A})=\frac{a_{4}+2\left(a_{3}-a_{2}\right)-a_{1}}{6}$,

$\operatorname{Ambiguity}(\tilde{A})=1, \operatorname{Ambiguity}(\tilde{B})=1.16, \operatorname{Ambiguity}(\tilde{C})=1.5$, and $\operatorname{Ambiguity}(\tilde{D})=1.5$.

Then, the value-Ambiguity interval approximation of $\tilde{A}$ is

$V_{A}(\tilde{A})=[\operatorname{Val}(\tilde{A})-A m b(\tilde{A}), \operatorname{Val}(\tilde{A})+A m b(\tilde{A})]$, which implies

$V_{A}(\tilde{A})=[4.3,6.3]$,

Similarly,

$V_{A}(\tilde{B})=[4,6.32], V_{A}(\tilde{C})=[3.3,6.3], V_{A}(\tilde{D})=[6,9]$.

Now, solve the above fuzzy game whose payoff matrix is given by

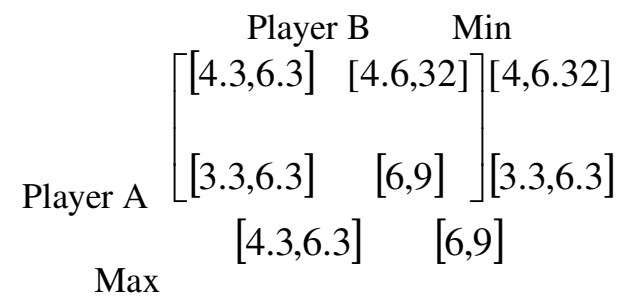

If Minmax $\neq$ Maximin 
Therefore the saddle point does not exist. Hence we have

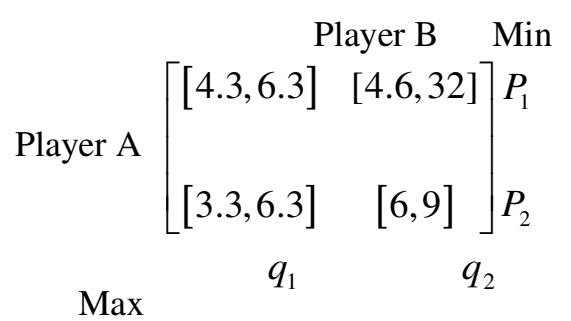

We know that,

Mixed strategy is followed. Moreover the value of the game is

$$
\begin{aligned}
\tilde{V} & =\frac{a_{11} a_{22}-a_{12} a_{21}}{\left(a_{11}+a_{22}\right)-\left(a_{12}+a_{21}\right)} \\
\tilde{V} & =\frac{[4.3,6.3][6,9]-[4,6.32][3.32,6.3]}{[[4.3,6.3]+[6,9]]-[[4,6.32]+[3.32,6.3]]}
\end{aligned}
$$

Therefore $\tilde{V}=5.19$

We know that,

$$
\begin{aligned}
& P_{1}=\frac{a_{22}-a_{21}}{\left(a_{11}+a_{22}\right)-\left(a_{12}+a_{21}\right)}=\frac{[6,9]-[3.3,6.3]}{(3,2.7)}=P_{1}=0.94 \\
& P_{1}+P_{2}=1 \Rightarrow P_{2}=1-P_{1}=0.06
\end{aligned}
$$

Also,

$$
q_{1}=\frac{a_{22}-a_{12}}{\left(a_{11}+a_{22}\right)-\left(a_{12}+a_{21}\right)}=\frac{[6,9]-[4,6.32]}{[3,2.7]}=0.82
$$

and $q_{1}+q_{2}=1 \Rightarrow q_{2}=1-q_{1}=0.18$

Therefore the value of the fuzzy game $(\tilde{V})=5.19$.

The Strategy for player A. i.e., $S_{A}=(0.94,0.06)=0.5$

Strategy for player B. i.e., $S_{B}=(0.82,0.18)=0.5$

\subsection{Distinct Approximation of Fuzzy Game without Saddle Point Method}

Let $\tilde{A}=(3,4,5,7), \tilde{B}=(2,5,6,7), \tilde{C}=(2,4,6,7), \tilde{D}=(4,7,8,11)$ are the trapezoidal fuzzy numbers and its Distinct approximation of Trapezoidal fuzzy number $\tilde{A}$ is

$$
\begin{aligned}
& D(\tilde{A})=\left(\frac{3 a_{1}-a_{2}}{2}, \frac{a_{1}+a_{2}}{2}, \frac{a_{3}+a_{4}}{2}, \frac{3 a_{4}-a_{3}}{2}\right) \text {. Using this, } D(\tilde{A})=(2.5,3.5,6,8), \\
& D(\tilde{B})=(1,3.5,6.5,7.5), D(\tilde{C})=(1,3,6.5,7.5), D(\tilde{D})=(2.5,5.5,9.5,12.5) .
\end{aligned}
$$

Now, solve the above fuzzy game whose payoff matrix is given by 
International Journal of Fuzzy Logic Systems (IJFLS) Vol.5, No.4, October 2015

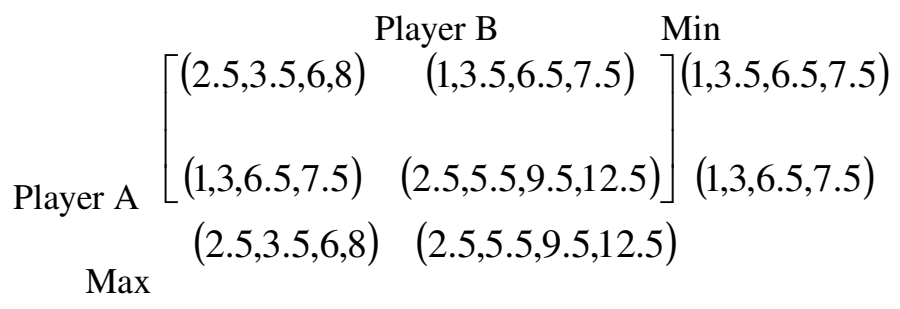

If Minmax $\neq$ Maximin

Therefore the saddle point does not exist.

Hence we have

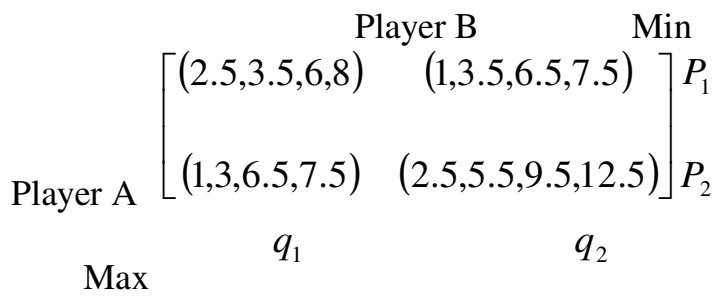

We know that,

Mixed strategy is followed. Moreover the value of the game is

$$
\begin{aligned}
& \tilde{V}=\frac{a_{11} a_{22}-a_{12} a_{21}}{\left(a_{11}+a_{22}\right)-\left(a_{12}+a_{21}\right)} \\
& \tilde{V}=\frac{[(2.5,3.5,6,8),(2.5,5.5,9.5,12.5)]-[(1,3.5,6 ., 7.5)(1,3,6.6,7.5)]}{[(5,9,15.5,20.5)]-[(2,6.5,13,15)]}=1.32
\end{aligned}
$$

We know that,

$$
\begin{aligned}
& P_{1}=\frac{a_{22}-a_{21}}{\left(a_{11}+a_{22}\right)-\left(a_{12}+a_{21}\right)}=0.22 \\
& P_{1}+P_{2}=1 \Rightarrow P_{2}=1-P_{1}=(0.78)
\end{aligned}
$$

Also, $q_{1}=\frac{a_{22}-a_{12}}{\left(a_{11}+a_{22}\right)-\left(a_{12}+a_{21}\right)}=0.21$

and $q_{1}+q_{2}=1 \Rightarrow q_{2}=1-q_{1}=(0.79)$

Therefore the value of the fuzzy game $(\tilde{V})=(1.32)$.

The Strategy for player A. i.e., $S_{A}=[(0.22,0.78)]=0.5$

Strategy for player B. i.e., $S_{B}=[(0.21,0.79)]=0.5$ 
International Journal of Fuzzy Logic Systems (IJFLS) Vol.5, No.4, October 2015

\section{Comparison Of Different ApProximations Of Fuzzy Numbers In Two-PERSon Zero Sum GaMe Method}

Here we have compared the different approximations of fuzzy numbers with its corresponding crisp number.

\begin{tabular}{|l|l|}
\hline & \multicolumn{1}{|c|}{ Example } \\
\hline 4.1 & The crisp number value of the game $(\tilde{V})=5.2$ \\
\hline 4.2 & The value of the game with the aid of trapezoidal fuzzy numbers $(\tilde{V})=5.2$ \\
\hline 4.3 & $\begin{array}{l}\text { The value of the game with the aid of close interval approximations of fuzzy numbers } \\
(\tilde{V})=4.7\end{array}$ \\
\hline 4.4 & $\begin{array}{l}\text { The value of the game with the aid of Value-Ambiguity interval Approximations of fuzzy } \\
\text { numbers }(\tilde{V})=5.19\end{array}$ \\
\hline 4.5 & The value of the game with the aid of Distinct Approximation of fuzzy numbers $(\tilde{V})=1.32$ \\
\hline
\end{tabular}

From the table it is noted that the value-ambiguity interval approximation is very close to the crisp number value of the game. Then we can conclude that the value-ambiguity interval approximation is much better than any other methods.

\section{CONCLUSION:}

In this paper, comparisons between different approximations of fuzzy numbers have been made by the aid of fuzzy game theory. The different approximations such as close interval, valueambiguity and distinct approximations have been employed in a fuzzy game are found and it is observed and concluded that the value-ambiguity approximation of fuzzy numbers is the best approximation than other methods. 


\section{REFERENCES}

[1] Billot .A., (1992) Economic Theory of Fuzzy Equilibria: An Axiomatic Analysis. ew York: SpringerVerlag.

[2] Butnariu .D.,( 1978) "Fuzzy Games: A Description of the Concept," Fuzzy Sets Syst., Vol. 1, Pp. 181-192.

[3] Eichberger.J., (1993) Game Theory for Economists. New York: Academic.

[4] Gillies.D.,(1953) "Locations of Solutions," Informal Conf. Theory of N-Person Games Princeton Mathematics, Princeton, $\mathrm{Nj}$, Pp. 11-12.

[5] Nash .J., (1950) "Equilibrium Points in N-Person Games," Proc. Nat. Academy Sci., New York, Ny, Vol. 36, Pp. 48-49.

[6] Nash .J., (1950) “The Bargaining Problem,” Econometrica, Vol. 18, Pp. 155-162,

[7] Shapley .L., (1953) "A Value for N-Person Games," Kuhn and Tucker, Eds., in Contributions to the Theory of Games. Princeton, Nj: Princeton Univ.Press, Pp. 207-317.

[8] Shapley .L., (1953) "Open Question," Informal Conf. Theory of N-Person Games Princeton Mathematics, Princeton, $\mathrm{Nj}, \mathrm{Pp} .11-12$.

[9] Stephen Dinagar .D and Jivagan .K., (2014). "A Note on Interval Approximation of Fuzzy Numbers", Proceedings of the International Conference on Mathematical Methods and Computation, ICOMAC.

[10] Stephen Dinagar .D and Jivagan .K., (2015) "Solving Transportation Problems using ValueAmbiguity interval Approximations of fuzzy numbers. International Journal of Recent Development in Engineering and Technology. (ISSN-2347-6435). Volume 2, Issue 5.

[11] Stephen Dinagar .D and Jivagan .K., (2015) Duality in Close Interval Approximation of Fuzzy Number Linear Programming. "Jamal Academic Research Journal (JARJ)" (ISSN No.:0973-0303). Proceedings of the International Conference on Mathematical Methods and Computation, ICOMAC, pp.674-681.

[12] Stephen Dinagar.D and Jivagan.K., (2015) "On Complement of Distinct Approximations of Fuzzy Number”. International Journal of Scientific Research and Engineering Trends. Volume 1, Issue 2, March-2015, ISSN (Online):2395- 566X.

[13] Von Neumann.J and Morgenstern.D., (1944). "The Theory of Games in Economic Bahavior. New York: Wiley.

[14] Zadeh.L.A., (1965) “Fuzzy Sets,” Inform. Contr., Vol. 8, Pp. 338-353.

[15] Zadeh.L.A., (1968), "Probability Measures And Fuzzy Events," J. Mathematics Anal.Applicat., Vol. 23, No. 2, Pp. 421-427. 